AUDIT

\title{
Providing treatment for hepatitis $C$ in an Australian district centre
}

\author{
A Ewart, L Harrison, B Joyner, A Safe
}

See end of article for

Postgrad Med J 2004;80:180-182. doi: 10.1136/pgmi.2003.005017 authors' affiliations

....................

Correspondence to:

Dr Amir Safe, Northern

General Hospital, Herries

Road, Sheffield S5 7AU,

UK; amirsafe@hotmail.

com

Submitted 5 January 2003

Accepted 11 March 2003
Hepatitis $\mathrm{C}$ virus (HCV) poses a major public health problem world wide. The introduction of combined therapy (interferon and ribavirin) and the recent development of pegylated interferon have offered the opportunity to alter the natural history of HCV, potentially reducing morbidity and mortality. Until recently, treatment has been confined to larger Australian cities. This paper describes the establishment of a clinic for the treatment of $\mathrm{HCV}$ in a regional Australian city. The facilities of the sexual health clinic were utilised. Factors contributing to the success of the clinic include the specialist nurse, a multidisciplinary approach, and the service model of shared care with general practitioners. The patient population and the outcomes of managing $\mathrm{HCV}$ in a regional centre are described. The sustained viral response rate is comparable to the published data from specialist centres.
C hronic hepatitis C virus (HCV) infection affects an estimated 170 million world wide, representing a pandemic. More than 200000 Australians are already infected and there are 11000 new cases per year mainly in young intravenous drug users. ${ }^{1}$ While hepatic cirrhosis, liver failure, and hepatocellular carcinoma occur in a minority, the major impact of the disease is in chronic ill health. HCV reduces patients' quality of life. ${ }^{2}$ A national HCV action plan was developed in 1994 and continues in the National Hepatitis C Strategy. ${ }^{3}$ The key components of this strategy include reducing the transmission of HCV in the community, treatment of HCV infection, and providing health care as well as support for people affected. The strategy aims to provide patients affected by HCV with equitable access to the full range of treatments. ${ }^{3}$ So far treatment has been confined mainly to the larger cities and there have been considerable difficulties in providing management for HCV outside state capital cities.

\section{ESTABLISHING A DISTRICT TREATMENT CENTRE}

By 2001 there were 700 notified HCV antibody positive tests in the catchment population of 150000 of the Rockhampton health district. Until 2000 no facility existed in Central Queensland for the treatment of HCV. In 1999, Rockhampton Hospital recruited a gastroenterologist who had previous experience in treating HCV with interferon. The liver clinic was established at Rockhampton in March 2000, utilising the facilities and expertise of a well established sexual health clinic. The clinic was called the "Liver Clinic" to reduce the stigma associated with HCV infection. There was no direct funding in Central Queensland for the treatment of HCV. However, the federal government funded pathology tests, as well as medications through the Pharmaceutical Benefits Scheme (section 100). The clinic was managed by reallocating resources within the sexual health budget.

\section{SHARED CARE MODEL}

A shared care model of service delivery was chosen to increase access to treatment, with limited specialist services. ${ }^{4}$ Educational activities were initiated to orientate general practitioners and hospital doctors to the availability of HCV therapy in the district. Information was provided to them by mail, through general practitioner newsletters, and by talks sponsored by the pharmaceutical industry.
The general practitioners were provided with a shared care package (table 1) and a supply of referral forms, that also detailed the investigations that should be performed by general practitioners before referral to the clinic. ${ }^{4}$ These include a HCV RNA test, as we would not offer therapy to someone with a negative test. Some patients who had negative tests were seen in the clinic for counselling.

There was a clear schedule of appointments with the clinic and general practitioner that was discussed with the patient (table 1).

\section{ROLE OF THE SPECIALIST NURSE}

It was appreciated that the management of HCV was complex and required a multidisciplinary approach. The keystone of the shared care model was the specialist nurse whose roles included:

- Liaising with all team members, patients and partners, general practitioners, psychologist, pharmacy, and professional HCV organisations.

- Providing education, counselling, and support to patients and carers during the treatment period.

- Arranging investigations and maintaining records and the clinic database.

- Supervising treatment, monitoring for side effects, and promoting contraception during treatment and for six months afterwards by both partners (ribavirin is teratogenic).

- Participating in consultations with medical practitioners.

\section{MANAGEMENT OF MENTAL HEALTH PROBLEMS}

The hospital psychologist reviewed all patients who were being considered for treatment to assess whether they would be able to cope with a complex treatment regimen, and to support the patients and carers with both pre-existing issues and problems that arose during the treatment period. Formal testing of anxiety and depression scales was undertaken. Patients with evidence or a history of psychosis were referred to a psychiatrist.

Abbreviations: $\mathrm{HCV}$, hepatitis $\mathrm{C}$ virus; $\mathrm{PCR}$, polymerase chain reaction 
Table 1 Interferon and ribavirin shared care schedule

\begin{tabular}{|c|c|c|c|c|c|c|c|c|c|c|c|c|c|c|c|c|c|c|c|c|}
\hline & \multicolumn{20}{|c|}{ Week } \\
\hline & 0 & 1 & 2 & 3 & 4 & 5 & 6 & 7 & 8 & 10 & 12 & 16 & 20 & 24 & 28 & 32 & 36 & 40 & 44 & 48 \\
\hline \multicolumn{21}{|l|}{ Interferon and } \\
\hline ribavirin prescription & $S p$ & & & & & & & & & & $S p$ & & & $S p$ & & & $S p$ & & & $S p$ \\
\hline Liver function tests & $S p$ & & $G P$ & & Sp & & & & $S p$ & & Sp & $S p$ & $S p$ & Sp & $S p$ & Sp & $S p$ & $S p$ & $S p$ & Sp \\
\hline Full blood count & $S p$ & $G P$ & $G P$ & $G P$ & $S p$ & & & & $S p$ & & Sp & Sp & Sp & $S p$ & $S p$ & $S p$ & $S p$ & Sp & Sp & Sp \\
\hline Thyroid function test & & & & & & & & & & & & up & up & up & & & & & & \\
\hline (TSH and thyroxine) & $S p$ & & & & & & & & & & $S p$ & & & $S p$ & & & $S p$ & & & $S p$ \\
\hline PCR & $S p$ & & & & & & & & & & & & & $S p^{*}$ & & & & & & $S p$ \\
\hline Lipids & $S p$ & & & & & & & & & & $S p$ & & & $S p$ & & & $S p$ & & & $S p$ \\
\hline $\begin{array}{l}\text { Urea and electrolytes } \\
\text { (including glucose and } \\
\text { urate) }\end{array}$ & $S p$ & & $G P$ & & $S p$ & & & & $S p$ & & $S p$ & $S p$ & $S p$ & $S p$ & $S p$ & $S p$ & $S p$ & $S p$ & $S p$ & $S p$ \\
\hline \multirow[t]{2}{*}{ Pregnancy test† } & $S p /$ & & & & Sp/ & & & & & & $S p$ & & $S p /$ & $S p$ & $S p /$ & Sp/ & $S p$ & $S p /$ & $S p /$ & $S p$ \\
\hline & $G P$ & & & & $G p$ & & & & $G P$ & & & $G P$ & $G P$ & & $G P$ & $G P$ & & $G P$ & $G P$ & \\
\hline Observationsł & $S p$ & $G P$ & $G P$ & $G P$ & Sp & & & & Sp & & $S p$ & $S p$ & $S p$ & $S p$ & $S p$ & $S p$ & $S p$ & $S p$ & $S p$ & $S p$ \\
\hline
\end{tabular}

\section{LIVER BIOPSY}

All patients who were eligible for treatment had a liver biopsy, unless contraindicated. The procedure was carried out as a day case according to a recommended protocol. ${ }^{5}$ Patients from outlying areas stayed in hospital overnight.

\section{COMBINATION THERAPY FOR HCV}

The clinic followed the Asia Pacific guidelines for treatment using the combined therapy of interferon and ribavirin (Rebetron, Schering-Plough). ${ }^{6}$ Interferon alfa $3 \mathrm{MU}$ is given three times weekly subcutaneously with oral ribavirin $1000 \mathrm{mg}$ daily for patients $<75 \mathrm{~kg}$ and $1200 \mathrm{mg}$ for patients $>75 \mathrm{~kg}$. HCV RNA was tested at six months. Patients with continuing positive results ceased treatment as did patients with genotype 3 who have responded. Responders with genotype 1 were offered a further six months of treatment. Pegylated interferon (Pegetron Schering-Plough) has been offered on a compassionate basis to non-responders for a six month course of combined therapy.

Treatment responses were recorded in the standard format: non-responder, end of therapy response, and sustained viral response PCR negative six months after stopping treatment).

\section{AUDIT RESULTS}

Information was extracted from the clinic database and supplemented by case notes review. Between March 2000 and October 2001, 73 people with HCV were referred and seen in the clinic. There were 36 men and 37 women. Sources of infections were similar to those reported from other

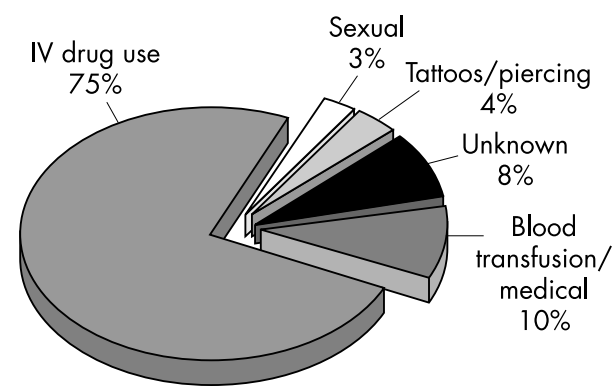

Figure 1 Sources of infection (\% of total); IV, intravenous.
Australian centres with $75 \%$ attributable to injecting drug use (fig 1). ${ }^{1}$ It was estimated that infection had been present for over 10 years in most patients $(70 \%)$ and none had been infected in the previous 12 months (fig 2). Twelve percent had a history of imprisonment or drug related offences. The HCV genotype was determined for 35 patients: 24 (69\%) had genotype 1 and $11(31 \%)$ had genotype 3 . The distribution was similar to that of other Australian studies. ${ }^{7}$ Of the referred patients, $34(46 \%)$ were eligible and offered treatment. This relatively high figure was probably due to the strict referral criteria.

Three patients were co-infected with HIV and HCV. None of these patients has been treated for HCV. One patient had documented poor adherence with HIV treatment, memory problems, social isolation, and alcohol abuse. Two patients declined treatment.

Twenty nine patients have received the combined therapy. Fourteen patients with genotype 1 received treatment. One patient had a sustained viral response and another had an end of therapy response. There were eight non-responders; two of these have been re-treated using pegylated interferon and ribavirin but failed to respond. Two patients failed to complete treatment. Of the 11 patients with genotype 3, five patients had a sustained viral response
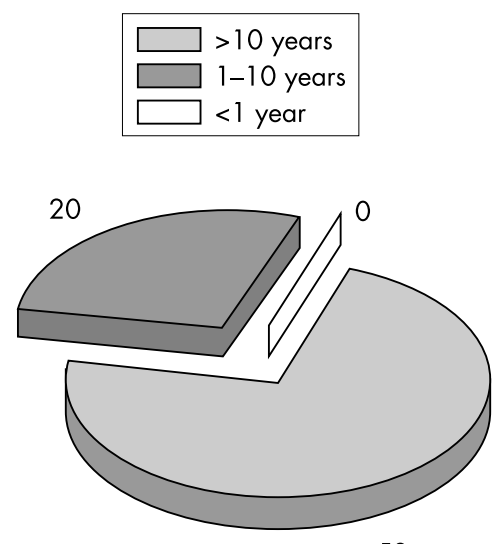

53

Figure 2 Time infected. 
and another two had an end of therapy response. Three patients are still on treatment and one patient ceased treatment because of depression. For five patients, the genotype was unknown, as genotyping was not required for the treatment regimen used when the clinic started. Four of these have sustained viral responses. One patient had treatment stopped because of abnormal liver function tests at 12 weeks according to the protocol at that time. Subsequent testing demonstrated genotype 1 and this patient has been offered re-treatment according to current protocols.

Overall, 25 of our 29 patients have now completed treatment with 10 sustained viral responses and three end of therapy responses. If these latter three patients remain PCR negative six months after treatment, there will be 13 sustained viral responses out of 25 treated patients and a response rate of $52 \%$ overall.

Ten patients required dose reductions of ribavirin due to haemolytic anaemia and two required dose reductions of interferon due to thrombocytopenia and neutropenia. One patient on pegylated interferon required dose reductions of ribavirin and interferon for anaemia and neutropenia respectively. One patient with established cirrhosis and thrombocytopenia was monitored closely and needed frequent platelet transfusion during six months of combined therapy. Two patients became hypothyroid during treatment and required thyroxine replacement. No patients developed a major side effect or complications from treatment.

One patient with a history of schizophrenia ceased treatment at his own request at week 10 and another patient ceased treatment at 12 weeks. One patient with genotype 1 ceased treatment after being PCR negative at 24 weeks. Seventeen $(59 \%)$ of the treated patients had a history of mental health problems and seven developed problems while on interferon. Three patients had a history of psychosis. They were referred for psychiatric assessment and a case manager was appointed to supervise them because of the risk of relapse on interferon therapy. Three patients (11\%) were already on antidepressant medications when treatment was started, and 12 (43\%) were started on treatment for depression during therapy. Sertraline was the main antidepressant drug used as its efficacy has been demonstrated. ${ }^{8}$

\section{DISCUSSION}

This audit demonstrates that, in the treatment of HCV, comparable outcomes to those achieved in tertiary centres ${ }^{9} 10$ were attained in this district centre with a multidisciplinary approach and a shared care programme. Most general practitioners cooperated with the programme, which sought to maintain continuity of care with the general practitioner. Close monitoring and effective communication facilitated adherence to the treatment regimen, timely adjustment of medications, and prompt management of side effects. General practitioners have been playing an active part in the patients' care particularly with arranging the initial necessary investigations and closely communicating with the specialist clinic for monitoring therapy.

Only three patients withdrew from treatment $(10 \%)$ and none developed any major complications. The sustained viral response in our unit was comparable to the published data from specialist centres. ${ }^{9}$ The results of treating genotype 1 were disappointing. However, there is evidence that the viral load responses translate into long term benefits. Japanese researchers have demonstrated that therapy potentially reduces the likelihood of late complications associated with HCV even in absence of sustained response. ${ }^{11}$

Informally, patients and general practitioners have been very satisfied with their local "Liver Clinic". There is now a local comprehensive treatment service that was previously available only in tertiary centres located hundreds of kilometres away. This "Liver Clinic" was established utilising the facilities of the sexual health clinic where staff already had significant knowledge and skills in the prevention and management of blood borne viruses, and were accustomed to dealing with issues of confidentiality and stigmatisation.

Two patients with genotype 1 who failed combined therapy were treated with pegylated interferon. Treatment with pegylated interferon results in a higher rate of response than does conventional monotherapy with interferon alfa. ${ }^{12}$ Large clinical trials are under way to evaluate the combination of pegylated interferon and ribavirin. Recent data showed that the use of pegylated interferon with ribavirin in genotype 1 patients gave higher sustained response than the use of interferon alfa and ribavirin. ${ }^{13}$ Currently patients who cannot be treated with ribavirin can be treated with pegylated interferon.

Comprehensive guidelines for the management of HCV are available. ${ }^{614}$ Interested specialists in district centres can provide standard care for these patients. Involvement in national audit programmes is important to ensure quality of care. The pharmaceutical industry has supported team training and there are national support group networks that provide high quality patient information.

There is an ongoing debate whether HCV infection should be managed in specialist (tertiary) centres or can be provided in district centres. Our audit supports the provision of treatment in district centres provided that there are interested clinicians, a specialist nurse, and an enthusiastic multidisciplinary team.

\section{Authors' affiliations}

A Ewart, A Safe, Department of Medicine, Rockhampton Base Hospital, Rockhampton, Queensland, Australia

L Harrison, Sexual Health Clinic, Rockhampton Base Hospital, Rockhampton, Queensland, Australia

B Joyner, Rural Clinical Division, University of Queensland, Rockhampton, Queensland, Australia

\section{REFERENCES}

1 Dore GJ, Pritchard-Jones J, Fisher D, et al. Who's at risk. Aust Fam Physician 1999:28(special issue):8-13.

2 Younissi ZM, Boparai N, Price LL, et al. Health-related quality of life in chronic liver disease: the impact of type and severity of disease. Am J Gastroenterol 2001;96:2199-205.

3 Commonwealth Department of Health and Aged Care. National hepatitis C strategy: 1999-2000 to 2003-2004. Canberra: Commonwealth Department of Health and Aged Care, 2000.

4 RACGP/ANCARD. Hepatitis C algorithms. Aust Fam Physician 1999;28(special issue):72-7

5 Grant A, Neuberger J. Guidelines on the use of liver biopsy in clinical practice. British Society of Gastroenterology. Gut 1999;45(suppl 4):Iv1-11.

6 Core Working Party for Asia-Pacific Consensus on Hepatitis B, C. Consensus statements on the prevention and management of hepatitis $B$ and hepatitis $C$ in the Asia-Pacific region. J Gastroenterol Hepatol 2000;15:825-41.

7 McCaw R, Moaven L, Locarini SA, et al. Hepatitis C virus genotype in Australia. J Viral Hepatol 1997;4:351-7.

8 Schramm TM, Lawford BR, Macdonald GA, et al. Sertraline treatment of interferon-alfa-induced depressive disorder. Med J Aust 2000;173:359-61.

9 Chander G, Sulkowski MS, Jenckes MW, et al. Treatment of chronic hepatitis C: a systemic review. Hepatology 2002;36(suppl):S135-44.

10 Barbaro G, Di Lorenzo G, Soldini M, et al. Evaluation of long-term efficacy of interferon alpha- $2 \mathrm{~b}$ and ribavirin in combination in native patients with chronic hepatitis C: an Italian multicenter experience. $J$ Hepatol 2000;33:482-4.

11 Nishiguchi S, Shiomi S, Nakatani S, et al. Prevention of hepatocellular carcinoma in patients with chronic hepatitis $C$ and cirrhosis. Lancet 2001;357:196-7.

12 Heathcote EJ, Shiffman ML, Cooksley WGF, et al. Peginterferon alfa 2a in patients with chronic hepatitis $C$ and cirrhosis. N EnglJ Med 2000;343:1673-80.

13 Di Bisceglie AM, Hoofragle JH. Optimal therapy of hepatitis C. Hepatology 2002;36(suppl 1):S121-7.

14 Booth JC, O'Grady J, Neuberger J. Clinical guidelines on the management of hepatitis C. Gut 2001;49(suppl 1):11-21. 